Original Article

\title{
Prevention of Sternal Wound Infections in Women Using an External Sternum Fixation Corset
}

\author{
Koen Selten, MD, Heike Schnoering, MD, PhD, Rashad Zayat, MD, PhD, Ali Aljalloud, MD,
} Ajay Moza, MD, PhD, Rüdiger Autschbach, MD, PhD, and Lachmandath Tewarie, MD

\begin{abstract}
Purpose: Deep sternal wound infection (DSWI) and mediastinitis are devastating complications after median sternotomy. Previous studies demonstrated an effective prevention of sternal wound infection (SWI) using an external sternal corset in high-risk cardiac surgery patients. The aim of this study is to assess the preventive effect of the Stern-E-Fix corset in high-risk poststernotomy female patients.

Methods: A total of 145 high-risk female patients undergoing cardiac surgery through median sternotomy were retrospectively analyzed. Patients were divided into group A $(n=71)$, who received the Stern-E-Fix corset (Fendel \& Keuchen GmbH, Aachen, Germany), and group B $(n=74)$, who received the elastic thorax bandage (SanThorax) postoperatively for 6 weeks. The mean follow-up period was 12 weeks.

Results: Incidence of SWI was $7 \%$ in group A vs. $17.6 \%$ in group B $(p=0.025)$. One patient presented with DSWI in group A vs. seven patients in group B $(p=0.063)$. No patient developed mediastinitis in group $A$ vs. four patients in group $B(p=0.121)$. In all, 4.2\% of group A patients required operative wound therapy vs. 16.2\% of group B patients $(p=0.026)$. The length of hospital stay was significantly longer in group $B(p=0.006)$.

Conclusion: Using an external supportive sternal corset (Stern-E-Fix) yields a significantly better and effective prevention against development of sternal dehiscence, DSWI, and mediastinitis in high-risk poststernotomy female patients.
\end{abstract}

Keywords: external sternal corset, sternal dehiscence, sternal wound infection, mediastinitis, Stern-E-Fix corset

Department of Thoracic and Cardiovascular Surgery, RWTH University Hospital, Aachen, Germany

Received: October 29, 2019; Accepted: March 24, 2020

Corresponding author: Koen Selten, MD. Department of Thoracic and Cardiovascular Surgery, RWTH University Hospital, Pauwelsstr. 30, 52074 Aachen, Germany

Email: kselten@ukaachen.de

Koen Selten and Heike Schnoering contributed equally to this study. *The abstract was presented at the 48th Annual Meeting of the German Society of Thoracic and Cardiovascular Surgery, April 2019, Wiesbaden, Germany.

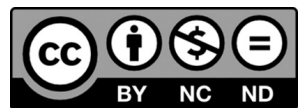

This work is licensed under a Creative Commons Attribution-NonCommercialNoDerivatives International License.

(C)2021 The Editorial Committee of Annals of Thoracic and Cardiovascular Surgery

\section{Introduction}

Deep sternal wound infection (DSWI) and mediastinitis are devastating complications after median sternotomy due to their association with greater morbidity and mortality. Short- and long-term outcomes of patients diagnosed with DSWI are worse, with an associated mortality rate reported between $10 \%$ and $47 \% .{ }^{1)}$ Despite improvement in prevention and treatment strategies, its incidence remains significant and ranges between $0.25 \%$ and $5 \%$. More than a century after introducing the median sternotomy, prevention and treatment of its complications remain a great challenge for cardiothoracic and plastic surgeons. ${ }^{1,2)}$ 
DSWI and mediastinitis are known to be a complex and multifactorial disease. Preoperative risk factors include diabetes mellitus (DM), obesity, advanced age, chronic obstructive pulmonary disease (COPD), smoking, female sex, acute or chronic renal failure, peripheral arterial disease (PAD), and emergent or urgent surgery. ${ }^{3)}$ Several preoperative risk factors have been identified, and of those, diabetes and obesity remain the most important risk factors. Intraoperative risk factors include the use of bilateral internal mammary arteries (BIMA), prolonged duration of surgery, cardiopulmonary bypass time and aortic crossclamp time, and redo cardiac surgery. Postoperative risk factors include postoperative respiratory failure and prolonged intensive care stay. The main postoperative risk factor is reoperation, especially for bleeding. ${ }^{3,4)}$

To prevent the complications after median sternotomy, many external stabilizers have been introduced. Some of them tend to be useful in preventing instability and infection of the sternum and should reduce postoperative pain, thereby improving the clinical course and the postoperative quality of life..$^{5-7)}$

Using the external supportive sternal Stern-E-Fix (Fendel \& Keuchen $\mathrm{GmbH}$, Aachen, Germany) corset is shown to yield a significantly better and effective prevention against development of sternal dehiscence and secondary sternal wound infection (SWI) in high-risk poststernotomy male patients. $\left.{ }^{6}\right)$

The aim of this study is to assess the prevention of SWI, DSWI, and mediastinitis after median sternotomy in high-risk female patients using the Stern-E-Fix corset.

\section{Materials and Methods}

In this retrospective study, 145 high-risk female patients who had cardiac surgery at our hospital from November 2009 to November 2012 were included. All female patients with a standard median sternotomy were included following informed consent. This study was carried out in compliance with the Declaration of Helsinki and approved by the local ethics committee. (EK 151/09)

All patients underwent different cardiac procedures of which $68 \%$ had coronary artery bypass grafting using standard median sternotomy, and all patients had equal preoperative preparations. The patients were divided into two consecutive groups: In group $\mathrm{A}, \mathrm{n}=71$ patients received a Stern-E-Fix corset for 6 weeks (Figs. 1A-1E). In group $\mathrm{B}, \mathrm{n}=74$ patients received a standard elastic thorax (SanThorax, Fendel \& Keuchen GmbH, Aachen, Germany) bandage for 6 weeks (Fig. 1F).
In both groups, conventional peri/trans-sternal wiring technique was used with eight stainless steel single wires (Ethicon Inc., Somerset, NJ, USA) or modified figure of eight. As part of our clinical standard, all patients in our department received on the first postoperative day (POD) an elastic sternal bandage (SanThorax) (group B), and since November 2009, we randomly started to use the new Stern-E-Fix corset (group A). All patients were evaluated on a daily routine. All patients were instructed on how to use the corset or bandage correctly. We advised all patients to wear the device until 6 weeks after sternotomy. We classified SWI and mediastinitis according to the guidelines of the Centers for Disease Control and Prevention (CDC) ${ }^{8)}$ and according to the El Oakley and Wright classification. ${ }^{4)}$

When an SWI was diagnosed, administration of appropriate antibiotics was based on culture and sensitivity results. In case of mechanical sternal dehiscence without clinical evidence of wound infection, patients were treated conservatively with the Stern-E-Fix corset (group A) or SanThorax elastic bandage (group B). The diagnosis of DSWI was based on clinical examination (fever, local infection signs, and fistulas), laboratory results (elevated levels of C-reactive protein and leukocytosis), or computed tomography (CT) scan (sternal dehiscence, retrosternal fluid collection). Microbiological examination was used in all cases to confirm the diagnosis. Prior to debridement and wound cleansing, wound cultures were taken. Immediate Gram staining and culture were done in two extra sets of wound swabs. In case of an evident mediastinal infection, culture- and sensitivity result-based appropriate antibiotics were administered. The antibiotic regimen and sterilizing technique were identical in both groups. The operation site was sterilized with standard povidone-iodine. In our department, all cardiac surgery patients routinely receive mupirocin nasal ointment the day before operation and perioperative prophylactic antibiotics with ampicillin/sulbactam (Unacid, Pfizer Pharma $\mathrm{GmbH}$, Berlin, Germany) or clindamycin in case of a penicillin allergy.

Postoperative daily wound care is based on a non-touch technique. Povidone-iodine wound cleansing is used. In case of a DSWI, operative wound debridement and vacuum-assisted wound therapy was performed in all patients. Wound and drain coverage was performed with a standard transparent adhesive bandage with a central absorbent pad. As part of our department clinical standard, all patients who developed SWI was followed up for 6 weeks after discharge through visits in our outpatient clinic to 

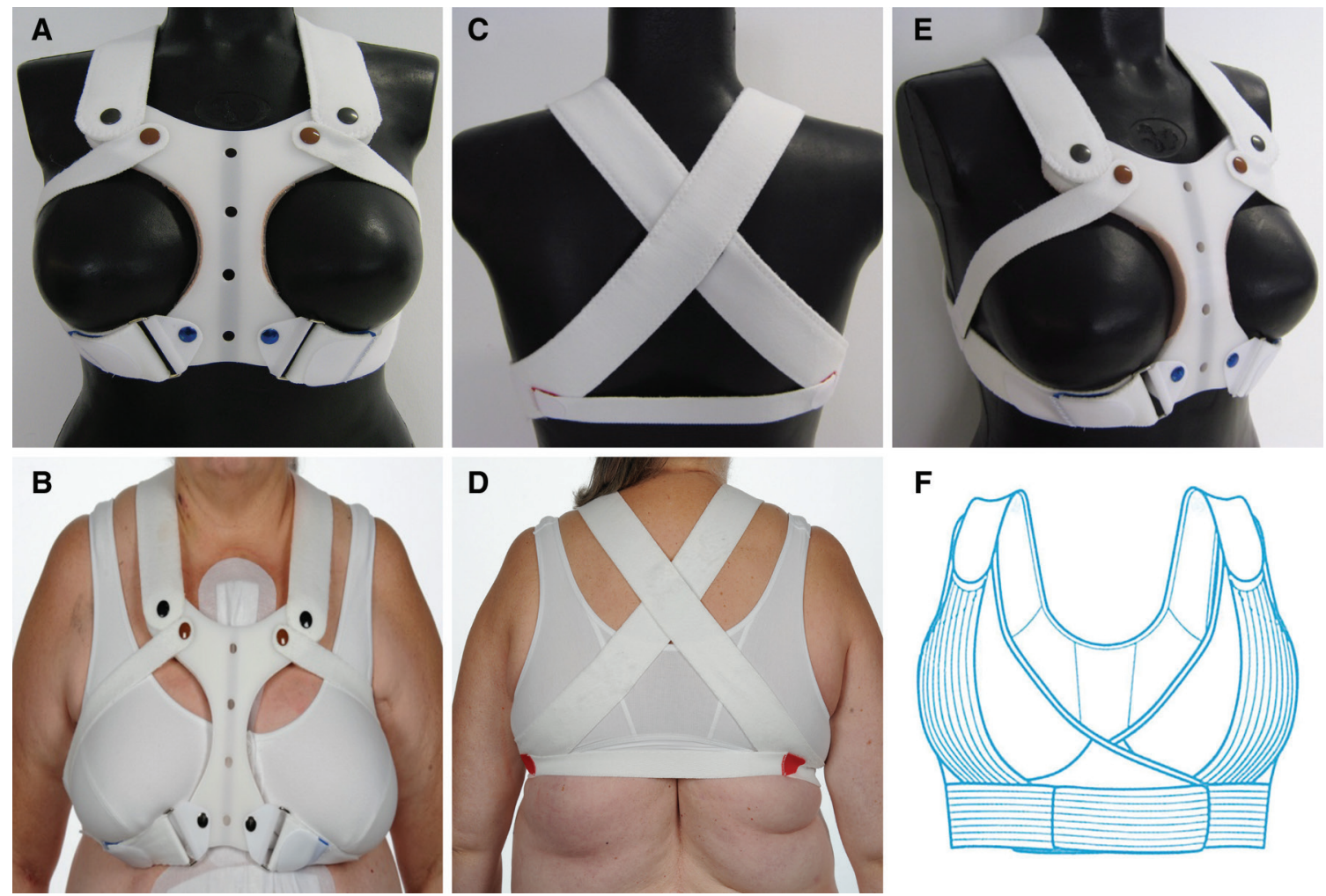

Fig. 1 (A-E) The Stern-E-Fix corset and (F) the SanThorax bandage: (A and B) anterior view of the Stern-E-Fix corset, (C and D) posterior view of the Stern-E-Fix corset, (E) anterolateral view of the Stern-E-Fix corset, and (F) anterior view of the SanThorax bandage.

control the sternal wound healing and clinical status. The mean follow-up period in this study was 12 weeks.

\section{Stern-E-Fix corset}

According to the manufacturer, the main goal was to make an anatomical and physical copy of the human thorax. The function of Stern-E-Fix corset is to prevent extreme chest expansion, without limiting the physical activity of the patient or respiratory muscles. It should promote postoperative rehabilitation, by reducing pain caused by physical strain on the chest. Furthermore, it must be easy to use for the patient and caregiver. In a poststernotomy thorax, excessive sternal overload can cause a variety of profound physically adverse effects that have the potential to lead to a significant increase in sternum instability, wound dehiscence, and secondary mediastinitis. $\left.{ }^{69}\right)$

The Stern-E-Fix corset consists of two parts as demonstrated in Figs. 1A-1E. The first part consists of a polyethylene front plate, in the form of sternum. The contact surface consists of a thin layer of silicon. This connection is the most important part of the corset. The second part consists of superior and inferior self-adhesive elastic bandages that follow the rib cage. Together with the front plate, the superior adhesive dressings prevent excessive movement of the rib cage in a forward direction and the inferior adhesive dressings prevent excessive movement of the rib cage in a lateral direction. Owing to the elastic flexibility of the Stern-E-Fix corset, patients are not limited during postoperative physical activities and during postoperative rehabilitation.

\section{Statistics}

Data analysis was performed with SPSS Statistics 24 (IBM, Chicago, IL, USA). Continuous variables were expressed as mean \pm standard deviation and categorical variables as absolute numbers and percentages. Continues variables were analyzed using the Student's $t$-test. Categorical variables were analyzed using the Chi-square test or, if appropriate, Fisher's exact test. All $p$-values were reported as three-digit numbers or with at least one non-zero digit. A $p$-value $<0.05$ was considered as statistically significant.

\section{Results}

Baseline characteristics of all patients are presented in Table 1. Distributions of known risk factors for the 
Table 1 Baseline characteristics and postoperative data

\begin{tabular}{lccc}
\hline Variables & $\begin{array}{c}\text { Stern-E-Fix } \\
\text { corset }(\mathrm{n}=71)\end{array}$ & $\begin{array}{c}\text { SanThorax } \\
\text { bandage }(\mathrm{n}=74)\end{array}$ & $p$-values \\
\hline Age, years & $71.5 \pm 9.6$ & $70.1 \pm 11.2$ & 0.374 \\
Female, n $(\%)$ & $71(100)$ & $74(100)$ & 1.000 \\
BMI, kg/m ${ }^{2}$ & $29.0 \pm 5.0$ & $28.5 \pm 4.6$ & 0.542 \\
Smoking, n (\%) & $10(14.1)$ & $18(24.3)$ & 0.143 \\
COPD, n $(\%)$ & $10(14.1)$ & $15(20.3)$ & 0.260 \\
NIDDM, n (\%) & $20(28.2)$ & $19(25.7)$ & 0.852 \\
IDDM, n (\%) & $7(9.9)$ & $10(13.6)$ & 0.608 \\
PAD, n $(\%)$ & $15(21.1)$ & $12(16.2)$ & 0.524 \\
Creatinine, mg/dl & $0.96 \pm 0.33$ & $0.90 \pm 0.30$ & 0.252 \\
Dialysis & 0 & 0 & 1.000 \\
NYHA >III, n $(\%)$ & $69(97.2)$ & $66(89.2)$ & 0.098 \\
CABG, n (\%) & $50(70.4)$ & $48(65.0)$ & 0.857 \\
Concomitant procedures, n (\%) & $18(25.4)$ & $20(27)$ & 0.852 \\
BIMA harvesting, n $(\%)$ & $2(2.8)$ & $3(4.1)$ & 1.000 \\
EuroSCORE II, \% & $5.6 \pm 4.6$ & $6.3 \pm 5.1$ & 0.385 \\
Bypass time, minutes & $121.6 \pm 51.1$ & $123.7 \pm 6.2$ & 0.294 \\
ICU stay, days & $6.3 \pm 5.9$ & $8.4 \pm 19.0$ & 0.154 \\
Pneumonia, n $(\%)$ & $22(30.9)$ & $15(20.0)$ & 0.133 \\
Septic shock & $3(4.2)$ & $3(4.0)$ & 0.999 \\
AKI & $9(12.7)$ & $6(8.0)$ & 0.419 \\
Re-sternotomy & $2(2.8)$ & $2(2.6)$ & 0.999 \\
Mechanical ventilation, days & $1.7 \pm 3.2$ & $2.0 \pm 3.5$ & 0.171 \\
Tracheostomy, n $(\%)$ & $3(4.2)$ & $2.5 \pm 3.9$ & 1.000 \\
PRBC & $1.7 \pm 1.5$ & 0.061 \\
\hline
\end{tabular}

BMI: body mass index $\left(\mathrm{kg} / \mathrm{m}^{2}\right)$; COPD: chronic obstructive pulmonary disease; NIDDM: non-insulin-dependent diabetes mellitus; IDDM: insulin-dependent diabetes mellitus; PAD: peripheral arterial disease; NYHA: New York Heart Association; CABG: coronary artery bypass grafting; BIMA: bilateral internal mammary artery; ICU: intensive care unit; AKI: acute kidney injury; PRBC: packed red blood cells

development of SWI were similar between the two groups (age, body mass index, COPD, smoking, diabetes, PAD, chronic renal failure, New York Heart Association classification, concomitant procedures, BIMA harvesting, Euroscore II, and cardio pulmonary bypass time). In both groups, the preoperative preparation and postoperative wound management were similar. The elastic bandage and the sternal corset were applied as soon as possible, mostly on first POD after surgery. All patients in both groups were regularly evaluated for signs and symptoms of delayed wound healing or wound infection during hospitalization. The postoperative data and outcomes are summarized in Table 1. The incidence of resternotomy in this study was equal $(n=2)$ in both groups. The mean duration of mechanical ventilation was comparable in both groups. Three patients in each group required percutaneous dilated tracheotomy due to prolonged mechanical ventilation.

Distribution of SWI is presented in Table 2. The overall incidence of SWI did differ significantly between the two groups in favor of the sternal corset group (group A: $7 \%[\mathrm{n}=5]$ vs. group B: $17.6 \%[\mathrm{n}=13], p=0.025)$. Patients treated with the Stern-E-Fix corset showed a tendency to have a lower incidence of DSWI (group A: $1.4 \%$ $[\mathrm{n}=1]$ vs. group B: $9.5 \%[\mathrm{n}=7], p=0.063)$. Mediastinitis did not occur in any patient in group A. On the contrary, four patients in group B $(p=0.120)$ did develop DSWI. There were three cases of mediastinitis related to mortality in group B. The SWI-related postoperative results are summarized in Table 3. The antibiotic therapy duration was significantly shorter in group A with $10 \pm$ 7.4 days vs. $20.2 \pm 15.5$ days in group $\mathrm{B}(p=0.001)$. Less surgical wound therapy and debridement were needed in group A than in group B (4.2\% vs. $16.2 \%$, respectively; $p=0.026$ ). The length of hospital stay (LOS) due to SWI was shorter in group A with $14.4 \pm 7.6$ days versus $22.3 \pm$ 15.8 days in group $\mathrm{B}(p=0.009)$. The overall LOS was also significantly shorter in group A with $15 \pm 9.2$ days versus $24.7 \pm 24.5$ days in group B $(p=0.006)$. 
Table 2 Distribution of sternal wound infection

\begin{tabular}{lccc}
\hline Variables & $\begin{array}{c}\text { Stern-E-Fix } \\
\text { corset }(\mathrm{n}=71)\end{array}$ & $\begin{array}{c}\text { SanThorax } \\
\text { bandage }(\mathrm{n}=74)\end{array}$ & $p$-values \\
\hline SWI, n (\%) & $5(7.0)$ & $13(17.6)$ & 0.025 \\
CDC I, n (\%) & $4(5.6)$ & $2(2.7)$ & 0.435 \\
CDC II, n (\%) & $1(1.4)$ & $7(9.5)$ & 0.063 \\
CDC III, n (\%) & 0 & $4(5.4)$ & 0.120 \\
\hline
\end{tabular}

SWI: sternal wound infection; CDC: centers for disease control and prevention

\section{Discussion}

The prevalence of multimorbid female patients undergoing cardiac surgery is progressively increasing not only in our institute but also worldwide. ${ }^{10-12)}$ In many previous studies, old, obese, diabetic female patients were found to have a significantly higher risk factor for developing DSWI after cardiac surgery. ${ }^{3,13,14)}$ In addition, they found obese diabetic patients to have a 2.1-fold greater risk of DSWI. This risk increased in the case of obese diabetic females by 1.6-fold due to the association of DM with obesity. ${ }^{15)}$ In patients with diabetes in our study group, we maintained glycemic control with insulin infusions in the early postoperative period and appropriately titrated doses in later phases of treatment. ${ }^{16)} \mathrm{We}$ strived early weaning from mechanical ventilation in all patients in our institution and especially in obese and high-risk patients and started early with mobilization and rehabilitation from the moment of awakening after surgery. These factors contribute to the reduction of respiratory complications and consequently may decrease sternal dehiscence rates. ${ }^{15,17)}$ In both of our study groups, no significant differences in mean mechanical ventilation and rehabilitation time were documented. As reported by Robicsek et al., ${ }^{18)}$ there is a direct relation between sternal dehiscence and the development of SWI. There are still differences of opinion in the literature about the operation-related risk factors such as prolonged heart lung machine time, duration of surgery, transfusion of red blood cells, use of double mammary artery, and use of pedicled or skeletonized mammary artery. ${ }^{19)}$ We found no significant difference in both study groups. The presence of comorbidities and life-threatening complications such as mediastinitis is associated with a 10\%-40\% mortality rate worldwide. In our study, three patients $(4 \%)$ died due to mediastinitis in group B and none in group A $(p=0.245)$.

Several biomechanical studies concluded that during excessive strain of sternum, the greatest separation of sternum takes place in the xiphoid than in the manubrium..$^{18,20,21)}$ Other studies suggest that sternal closure techniques using osteosynthesis plates and wires probably make little or no difference regarding the prevention of sternal complications in the postoperative period when compared to the standard closure method in adults, regardless of gender and race. ${ }^{22)}$ In our study group, a similar surgical wound closure technique was used, eight transsternal or peristernal single steel wires (Ethicon Inc., Somerset, NJ, USA) followed by multiple layer sutures. Infection of the operative wound and sternal dehiscence are two closely associated complications, although it is rarely possible to determine which one causes the other. During postoperative rehabilitation, an increased risk of sternum overload is not uncommon ${ }^{23,24)}$; therefore, external devices such as a sternal corset is recommended for the prevention of sternal dehiscence and secondary prevention of SWI. Such a viable instrument against sternal stress forces was demonstrated in group A, which showed an extremely low incidence of sternal dehiscence.

Multimorbid women in both patient groups were unable to take care of themselves, which led to an insufficient compliance with regard to mobilization, use of medication, and general daily care. This ultimately led to incorrect and insufficient postoperative rehabilitation. This group of patients was regularly monitored on an outpatient basis to prevent postoperative complications. ${ }^{23)}$ The extent of sternal dehiscence, DSWI, and mediastinitis cannot be made on chest radiography only. The main postoperative strategy in our study group was once sternal dehiscence and SWI became evident, CT was performed. ${ }^{24,25)}$ In our study group, we did not find any patients with sternal dehiscence after 12 weeks. Besides sternal precautions, we did not recommend our patients further treatment with Stern-E-Fix corset after 12 weeks. The economic impact of postoperative mediastinitis has also been evaluated in several studies. ${ }^{26-28)}$ Patients with mediastinitis were shown to be hospitalized 20 days longer than patients without this complication. 
Table 3 Sternal wound infection-related outcomes

\begin{tabular}{lccc}
\hline Variables & $\begin{array}{c}\text { Stern-E-Fix } \\
\text { corset }(\mathrm{n}=71)\end{array}$ & $\begin{array}{c}\text { SanThorax } \\
\text { bandage }(\mathrm{n}=74)\end{array}$ & $p$-values \\
\hline Conservative therapy & $2(2.8)$ & $1(1.4)$ & 0.610 \\
Operative wound therapy & $3(4.2)$ & $12(16.2)$ & $\mathbf{0 . 0 2 6}$ \\
SWI recurrence & 0 & $2(2.7)$ & 0.497 \\
Antibiotics therapy, days & $10 \pm 7.4$ & $20.2 \pm 15.5$ & $\mathbf{0 . 0 0 1}$ \\
Overall LOS, days & $15 \pm 9.2$ & $24.7 \pm 24.5$ & $\mathbf{0 . 0 0 6}$ \\
LOS due to SWI, days & $14.4 \pm 7.6$ & $22.3 \pm 15.8$ & $\mathbf{0 . 0 0 9}$ \\
POD of SWI diagnosis & $1.3 \pm 5.5$ & $5.6 \pm 20.1$ & 0.080 \\
Overall mortality & $2(2.8)$ & $4(5.3)$ & 0.681 \\
Mortality due to SWI & 0 & $3(4.0)$ & 0.245 \\
\hline
\end{tabular}

SWI: sternal wound infection; LOS: length of stay; POD: postoperative day

Patients in our study were shown to have a significantly reduced hospitalization in the Stern-E-Fix group. The main findings of this study are the reduction of sternal dehiscence and a tendency to reduce DSWI and mediastinitis by using the Stern-E-Fix corset. Since then, we have continued to use the Stern-E-Fix corset in all our poststernotomy patients.

\section{Limitations}

Even though our data demonstrate an effect of the Stern-E-Fix corset on reducing the incidence of DSWI, our study is limited by the usual shortcomings of a small-cohort, single-centre study. One other limitation of our study is that the data suffer potential bias in patient selection and data acquisition. SWI after sternotomy is multifactorial, and as a result, we cannot exclude the possibility that unmeasured confounders influenced our results.

Therefore, a further prospective and randomized study to analyze larger number of patients is encouraged to be able to detect a clear preventive effect of an external sternal corset.

\section{Conclusion}

In prevention of sternal dehiscence and SWIs, it is important to keep focus on every detail in peri- and postoperative rehabilitation care. The outcome of this study shows that using an external supportive sternal corset (Stern-E-Fix) provides a significant better and effective prevention against development of sternal dehiscence and a tendency to better prevention against development of DSWI and mediastinitis in high-risk poststernotomy female patients undergoing cardiac surgery.

The Stern-E-Fix corset was also found to reduce the need for operative therapy and to reduce the overall length of hospitalization and the length of hospitalization due to SWI.

\section{Disclosure Statement}

All authors have no conflict of interest.

\section{References}

1) Abu-Omar Y, Kocher GJ, Bosco P, et al. European Association for Cardio-Thoracic Surgery expert consensus statement on the prevention and management of mediastinitis. Eur J Cardiothorac Surg 2017; 51: 10-29.

2) Yusuf E, Chan M, Renz N, et al. Current perspectives on diagnosis and management of sternal wound infections. Infect Drug Resist 2018; 11: 961-8.

3) Meszaros K, Fuehrer U, Grogg S, et al. Risk factors for sternal wound infection after open heart operations vary according to type of operation. Ann Thorac Surg 2016; 101: 1418-25.

4) El Oakley RM, Wright JE. Postoperative mediastinitis: classification and management. Ann Thorac Surg 1996; 61: 1030-6.

5) Caimmi PP, Sabbatini M, Kapetanakis EI, et al. A randomized trial to assess the contribution of a novel thorax support vest (corset) in preventing mechanical complications of median sternotomy. Cardiol Ther 2017; 6: 41-51.

6) Tewarie LS, Menon AK, Hatam N, et al. Prevention of sternal dehiscence with the sternum external fixation (Stern-E-Fix) corset--randomized trial in 750 patients. J Cardiothorac Surg 2012; 7: 85.

7) Gorlitzer M, Wagner F, Pfeiffer S, et al. Prevention of sternal wound complications after sternotomy: results of a large prospective randomized multicentre trial. Interact Cardiovasc Thorac Surg 2013; 17: 515-22.

8) Horan TC, Gaynes RP, Martone WJ, et al. CDC definitions of nosocomial surgical site infections, 1992: a modification of CDC definitions of surgical 
wound infections. Am J Infect Control 1992; 20: 271-4.

9) Irwin RS. Complications of cough: ACCP evidence-based clinical practice guidelines. Chest 2006; 129: $54 \mathrm{~S}-58 \mathrm{~S}$.

10) Wiedemann D, Bernhard D, Laufer G, et al. The elderly patient and cardiac surgery - a mini-review. Gerontology 2010; 56: 241-9.

11) Pierri MD, Capestro F, Zingaro C, et al. The changing face of cardiac surgery patients: an insight into a Mediterranean region. Eur J Cardiothorac Surg 2010; 38: 407-13.

12) Ridderstolpe L, Gill H, Granfeldt H, et al. Superficial and deep sternal wound complications: incidence, risk factors and mortality. Eur J Cardiothorac Surg 2001; 20: 1168-75.

13) Hussey LC, Hynan L, Leeper B. Risk factors for sternal wound infection in men versus women. Am J Crit Care 2001; 10: 112-6.

14) Thelwall S, Harrington $P$, Sheridan E, et al. Impact of obesity on the risk of wound infection following surgery: results from a nationwide prospective multicentre cohort study in England. Clin Microbiol Infect 2015; 21: 1008.e1-8.

15) Bonacchi M, Prifti E, Bugetti M, et al. Deep sternal infections after in situ bilateral internal thoracic artery grafting for left ventricular myocardial revascularization: predictors and influence on 20-year outcomes. J Thorac Dis 2018; 10: 5208-21.

16) Szabó Z, Hakanson E, Svedjeholm R. Early postoperative outcome and medium-term survival in 540 diabetic and 2239 nondiabetic patients undergoing coronary artery bypass grafting. Ann Thorac Surg 2002; 74: 712-9.

17) Robicsek F, Daugherty HK, Cook JW. The prevention and treatment of sternum separation following openheart surgery. J Thorac Cardiovasc Surg 1977; 73: 267-8.
18) Robicsek F, Fokin A, Cook J, et al. Sternal instability after midline sternotomy. Thorac Cardiovasc Surg 2000; 48: 1-8.

19) Fokin AA, Robicsek F, Masters TN, et al. Sternal nourishment in various conditions of vascularization. Ann Thorac Surg 2005; 79: 1352-7.

20) Cohen DJ, Griffin LV. A biomechanical comparison of three sternotomy closure techniques. Ann Thorac Surg 2002; 73: 563-8.

21) McGregor WE, Trumble DR, Magovern JA. Mechanical analysis of midline sternotomy wound closure. J Thorac Cardiovasc Surg 1999; 117: 1144-50.

22) Cataneo DC, Dos Reis TA, Felisberto G, et al. New sternal closure methods versus the standard closure method: systematic review and meta-analysis. Interact Cardiovasc Thorac Surg 2019; 28: 432-40.

23) Fortin M, Dubois MF, Hudon C, et al. Multimorbidity and quality of life: a closer look. Health Qual Life Outcomes 2007; 5: 52.

24) Bitkover CY, Cederlund K, Aberg B, et al. Computed tomography of the sternum and mediastinum after median sternotomy. Ann Thorac Surg 1999; 68: 858-63.

25) El-Ansary D, Waddington G, Adams R. Measurement of non-physiological movement in sternal instability by ultrasound. Ann Thorac Surg 2007; 83: 1513-6.

26) Sears ED, Wu L, Waljee JF, et al. The impact of deep sternal wound infection on mortality and resource utilization: a population-based study. World J Surg 2016; 40: $2673-80$.

27) Diez C, Koch D, Kuss O, et al. Risk factors for mediastinitis after cardiac surgery - a retrospective analysis of 1700 patients. J Cardiothorac Surg 2007; 2: 23.

28) Hollenbeak CS, Murphy DM, Koenig S, et al. The clinical and economic impact of deep chest surgical site infections following coronary artery bypass graft surgery. Chest 2000; 118: 397-402. 